\title{
Recovery of Myocardial Hibernation after Percutaneous Coronary Intervention
}

\section{Repetitive Assessment by Magnetic Resonance Imaging \\ Frank Breuckmann', Kai Nassenstein², Jörg Barkhausen², Gerd Heusch ${ }^{3}$, Raimund Erbel ${ }^{1}$}

Rahimtoola first proposed the pathophysiological concept of myocardial hibernation to characterize a situation of a prolonged subacute or chronic state of myocardial ischemia in which myocardial contractility, ventricular function, and metabolism are changed to match the reduced blood supply [1].

Hibernating myocardium is characterized by regional contractile dysfunction in noninfarcted myocardium in the setting of a severe coronary stenosis. Whenever critical regional wall impairment is detected in the absence of an active or former occlusion of the corresponding coronary vessel, myocardial hibernation has to be taken into account. In contrast to infarcted myocardium, hibernating myocardium has impaired contractile function and improves after revascularization [2]. Therefore, the detection of myocardial hibernation in the absence of myocardial necrosis or fibrosis is important for further therapeutic options, e.g., percutaneous coronary intervention (PCI) or coronary artery bypass graft.

Recently, using cardiovascular magnetic resonance perfusion imaging, Selvanayagam et al. demonstrated that hibernating myocardial segments have significantly lower resting myocardial blood flow than remote myocardial segments [3]. Apart from first-pass perfusion imaging, fast low angle shot (FLASH) sequences directly following infusion of contrast agent may also be used to visualize blood flow.

Our image series is considered a typical example of myocardial hibernation (Figures $1 \mathrm{a}$ and $1 \mathrm{~b}$ ) and its resolution after PCI (Figures 1c and 1d), underlining the impact of noninvasive imaging in the decision process of planning adequate therapy.

\section{References}

1. Rahimtoola SH. A perspective on the three large multicenter randomized clinical trials of coronary bypass surgery for chronic stable angina. Circulation 1985;72:Suppl V:V-123-35.

2. Heusch G, Schulz R, Rahimtoola SH. Myocardial hibernation: a delicate balance. Am J Physiol Heart Circ Physio 2005;288:H984-99.

3. Selvanayagam JB, Jerosch-Herold $M$, Porto I, et al. Resting myocardial blood flow is impaired in hibernating myocardium: a magnetic resonance study of quantitative perfusion assessment. Circulation 2005;112:3289-96.

\section{Korrespondenzanschrift}

Dr. Frank Breuckmann

Universitätsmedizin der Johannes Gutenberg-Universität Mainz

Langenbeckstraße 1

55131 Mainz

Telefon (+49/6131) 17-5737, Fax -566o

E-Mail: grasmueck@2-med.klinik.uni-mainz.de
II. Medizinische Klinik und Poliklinik tion as a sign of periinterventional microembolization (d).
Department of Cardiology, West German Heart Center Essen, Germany,

${ }^{2}$ Department of Diagnostic and Interventional Radiology and Neuroradiology, University of DuisburgEssen, Essen, Germany, ${ }^{3}$ Institute of Pathophys iology, University of Duisburg-Essen, Essen, Germany.

Herz 2009:34:240-2 DOI $10.1007 /$ s00059-009-3229-2

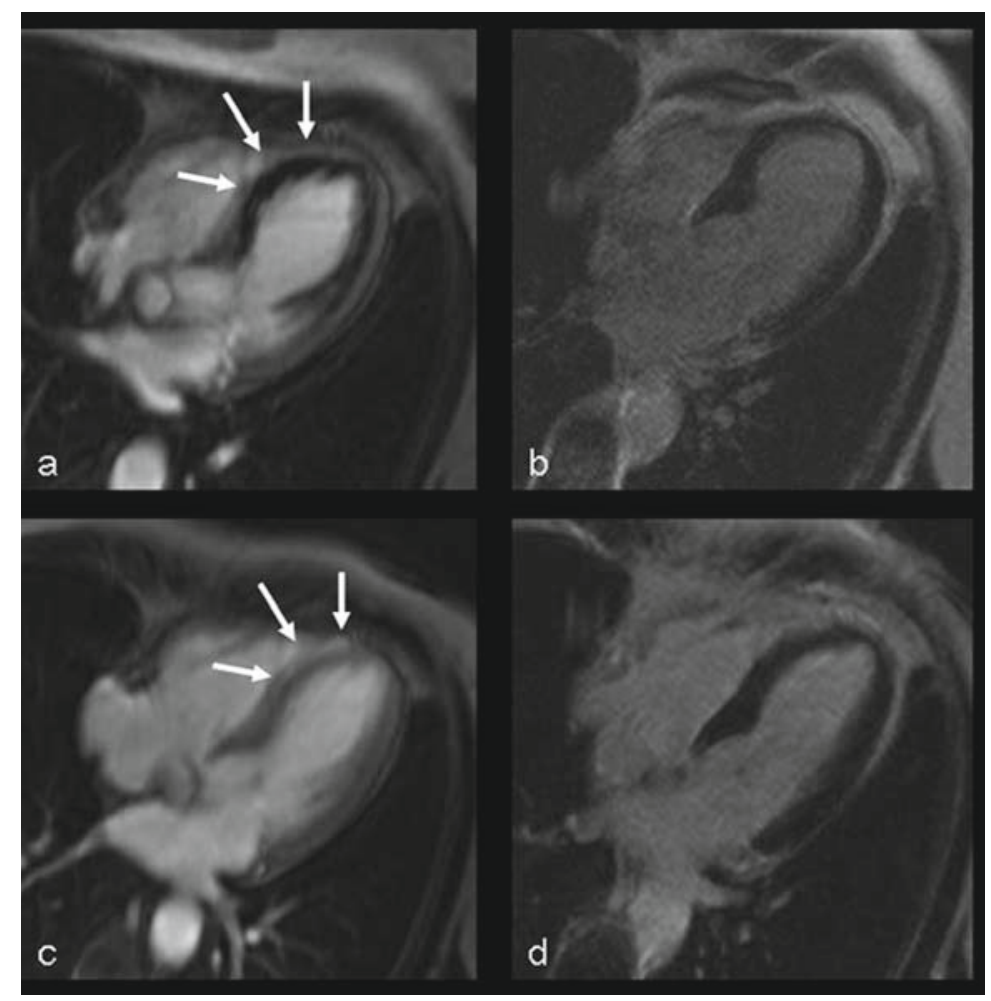

Figures 1a to 1d. Long axis views. Preinterventional resting subendocardial ischemia (a, white arrows) in FLASH sequences is observed in the septal and apical wall without concomitant late enhancement (LE; $b$ ). Due to the absence of $L E$ as a sign of irreversible myocardial damage, $\mathrm{PCl}$ of the left main stem and the left anterior descending artery was performed. 1 week after the intervention, resting subendocardial ischemia (c, white arrows) was completely resolved without new LE forma- 